\title{
Pedestrian detection via fusional convolutional features
}

\author{
Yang Dongming ${ }^{*}, 1,2$, Ge Shuiying ${ }^{1}$ \\ ${ }^{1}$ National Science Library of CAS, Beijing 100090 \\ 2.University of Chinese Academy of Sciences, Beijing 100049, China \\ 475527783@qq.com
}

Keywords: Pedestrian detection, Convolutional network, Multiscale, Fusional features.

\begin{abstract}
The pedestrian detection methods based on convolutional networks showed a more advantage than methods with artificial features. However, most algorithms do not achieve the desired results because they have not made full use of convolutional features. In this paper, we proposed a model called Multiple Fused CNNs, which utilizes fusional feature maps from multi-scale convolutional layers to detect pedestrians.
\end{abstract}

\section{Introduction}

Pedestrian detection technology has a wide range of applications such as auxiliary driving and intelligent monitoring. However, most pedestrian detection methods are infeasible to be opted into practical systems because of the poor robustness in a variety of scenarios. Therefore, a model with simple structure, which can perform better accuracy with fast speed, will be welcomed by practical applications.

\section{Related work}

The initial methods of artificial features based pedestrian detection, whose accuracy depends on the quality of the feature and classifier mostly. There are plenty of impressive artificial features such as HOG[1] LBP[2] and ACF[3]. These artificial features still play critical roles in some state-of-the-art pedestrian detection methods. Before 2015, most pedestrian detection methods are focus on artificial features, such as Katamari[4], which achieved 22.49\% MR on Caltech[20] dataset by using HOG+Flow features. However, these methods have limited space for improvement.

Since 2012, CNNs has been utilized for objects detection and has become the mainstream. Thus, some paper like [5][6][7] use CNNs instead of artificial methods to extract pedestrian features. Some innovative work has been done, DeepParts[11] improved performance by handling occlusion with an extensive part pool for instance. Unfortunately, these models are very slow with complex structures.

On the other hand, methods based on region proposal CNNs are very popular. R-CNN (Regions with CNNs)[8] combines selective search region proposals and CNNs based post-classification. Fast R-CNN[9] uses ROI(region of interest) pooling layer and minimizing loss for both confidences and bounding box regression. Faster R-CNN[10] use RPN (Regions Proposal Network) to replaces selective search proposals and integrate RPN with Fast R-CNN.

Nevertheless, the R-CNN based methods has a obvious flaw: They detect objects over a single feature map. Although the framework has achieved a good performance after being improved, but it is still not good at multi-scale objects detection, especially low-resolution objects. 
$\mathrm{RPN}+\mathrm{BF}[12]$ overcomes the limitations of Faster R-CNN by utilizing BF (Boosted Forest) to reuse the deep convolutional features of RPN, which achieve an amazing result, but it is not fast enough.

Besides, there are some end-to-end model such as YOLO[13], which has a great advantage on efficiency but doesn't have a proud recall rate. HyperNet[15], ParseNet[16] and ION[17] combine features from multiple feature maps. FPN (Feature Pyramid Network)[18] use lateral connections to connect multiple feature maps with a top-down architecture for building high-level semantic feature maps at all scales. These models work well but are at the expense of speed.

This paper follows the idea of $\operatorname{SSD}[14]$ to propose a pedestrian detector based on multiple convolutional feature maps, the difference is we fuse the multi-scale feature maps before detecting, which can make full use of multi-scale semantic information from different feature maps.

Our obvious contributions include:

- We proposed a method for fusing convolutional features from different layers. This method improved accuracy of detection.

-We put our model into SSD framework and applied it to pedestrian detection. The results showed that the model performed very well.

-We compared our model with other state-of-the-arts of pedestrian detection and showed good competitiveness.

\section{Method}

Model Structure. Traditional algorithms build features pyramid by extracting artificial feature over dense image pyramid like Figure 1, which is very slow. Faster R-CNN utilized a set of multi-scale anchor boxes in RPN to provide multiple proposal, but these proposal boxes do not work over multi-scale feature maps. Thus, Faster R-CNN misses the rich semantic information from different feature maps. There is a model of fused feature maps called SharpMask, which also detects over a single feature map.
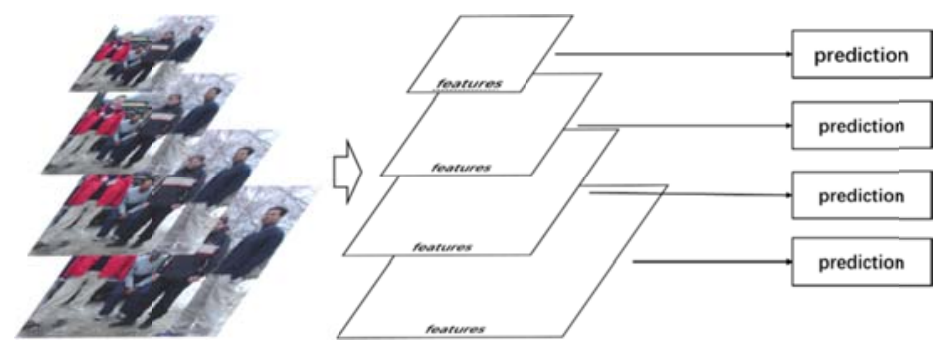

Figure 1 (a) Traditional algorithms use features pyramid for detection by extracting artificial feature over dense image pyramid
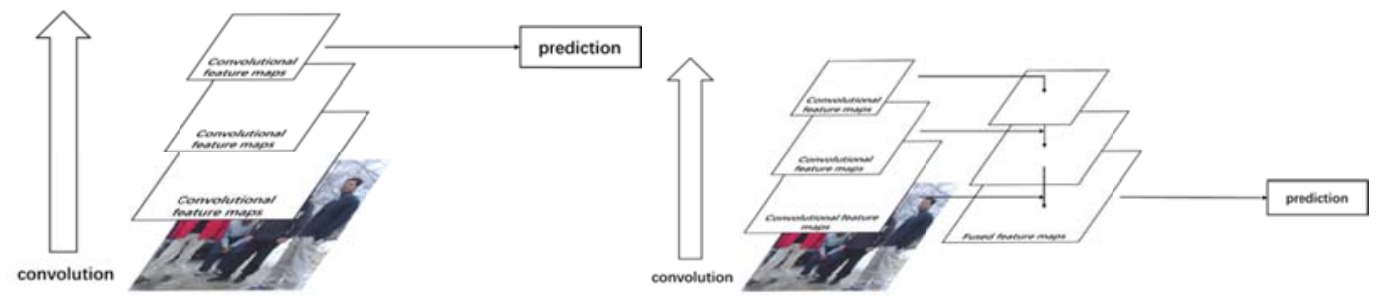

$\begin{array}{lll}\text { (b) Faster R-CNN uses a single feature map for detection } & \text { (c) SharpMask uses a single fused }\end{array}$ feature map for detection

In this paper, we use a set of bounding boxes from several fused feature maps with different resolutions to detect pedestrians, which achieves a better accuracy. 


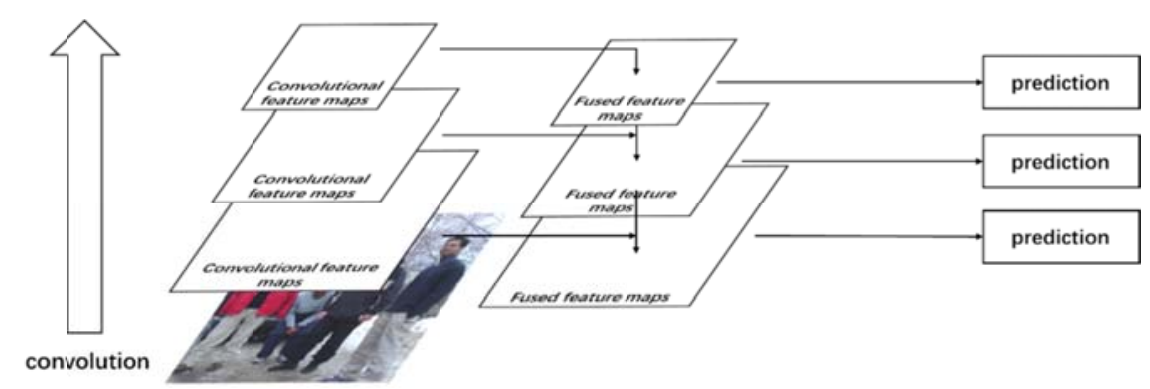

Figure 2 Our model uses multiscale fused feature maps for idetection

Fusional layers. Feature maps from different convolutional layers have different resolution and semantic information. The shallow layers create feature maps with high resolution but with weak semantics. These maps are not robust and provide unsatisfactory prediction. The higher-level feature maps are semantically strong but lack of location information because of the low resolution. Our model improves the detection performance by combining the advantages of all these multiple layers.

Shown in Figure 3, we feed the model with the image of $512 * 512 * 3$. We use the atrous[19] version of a subsampled VGG16 as the basic network and adds several convolutional layers then. The added layers are $\{M 1, M 2, M 3, M 4, M 5, M 6, M 7\}$, they have different resolution of $\{64 *$ $64,32 * 32,16 * 16,8 * 8,4 * 4,2 * 2,1 * 1\}$. Fused feature maps are $\{\mathrm{F} 1, \mathrm{~F} 2, \mathrm{~F} 3, \mathrm{~F} 4, \mathrm{~F} 5, \mathrm{~F} 6\}$, which have the same resolution with $\mathrm{M} 1 \sim \mathrm{M} 6$. We finally use F1 F6 and M7 for detection.

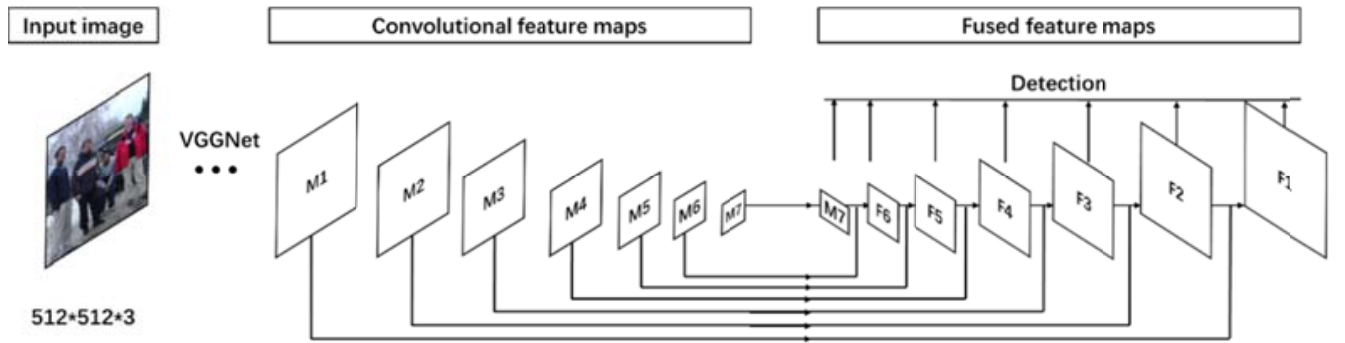

Figure 3 Our model uses multiscale fused feature maps for idetection

Details of feature fusion. Figure 4 shows an example of feature fusion. Because of the different resolution of these convolutional feature maps, the first job is upsampling the smallest feature map (M7) by a factor of 2 to make it as big as M6. The most common implementation of upsampling is using interpolation. Nearest Neighbor Interpolation is the easiest way that using the nearest original pixel to assign to the new pixel.

Feature maps from different convolutional layers have different number of channels. For instance, VGG16 has five sets of convolutional operations, the channels of output feature maps are $\{64,128,256,512,512\}$. In order to fuse the maps from different layers, we append $1 \times 1$ convolutions on maps to unify the number of channels to 256 .

Finally, we can merge two feature maps by addition. In order to reduce the influence of feature aliasing, we append a $3 \times 3$ convolution after each fusion. Finally, we obtain six fused feature maps.

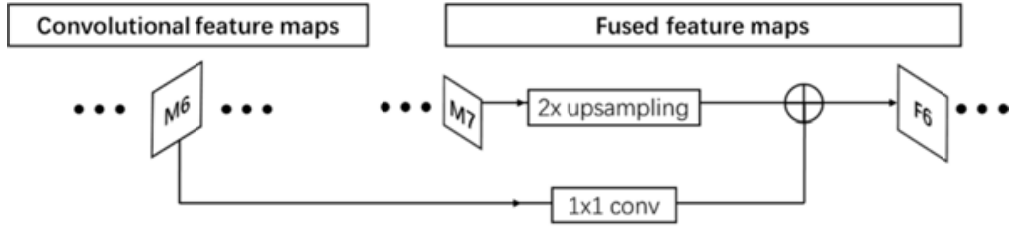

Figure 4 An example of feature fusion 


\section{Experimental results}

The experiment is running based on SSD network structure. The evaluation dataset is based on Caltech pedestrian Detection Benchmark [20], which includes about 250,000 frames (in 137 approximately minute long segments) with a total of 350,000 bounding boxes. About 2300 unique pedestrians were annotated. We extract a frame every five frames from Caltech training dataset and all frames from ETH dataset and TUD-Brussels dataset for training while 4024 images in the standard test set are used for evaluation.

We fine-tune the model from VOC Pascal 2007 and 2012. The initial learning rate is set to 0.001 . We use SGD (Stochastic Gradient Descent) to fine-tune the model while momentum is set to 0.9. We set the maximum iteration number as 40,000 .

Comparison with State-of-the-Arts. Table 1 shows the result of our model compared with state-of-the-art models on Caltech dataset. The evaluation metric is log-average Miss Rate on False Positive Per Image (FPPI) in $\left[10^{-3}, 10^{1}\right]$. Our model has an MR of $12.05 \%$ while the best result from $\mathrm{RPN}+\mathrm{BF}$ is $9.58 \%$. Although our model is not the best in this evaluation, it is the most competitive one on speed. In addition, not like most methods, we use no artificial features but just a single convolutional network for detection.

Table 1. Comparison with other methods on Caltech set using a default IoU threshold of 0.5 to determine True Positives.

\begin{tabular}{c|c}
\hline Method & MR \\
\hline RPN+BF & $\mathbf{0 . 0 9 5 7 7 3}$ \\
\hline CompACT-Deep & 0.117475 \\
\hline Deep Parts & 0.118890 \\
\hline Multiple Fused CNNs (ours) & 0.120456 \\
\hline Checkerboards + & 0.170986 \\
\hline CCF+CF & 0.173247 \\
\hline Checkerboards & 0.184720 \\
\hline CCF & 0.187086 \\
\hline TA-CNN & 0.208573 \\
\hline SCCPriors & 0.218599 \\
\hline
\end{tabular}

Performance on scale and occlusion subsets. For existing pedestrian detection system, the ability of handling multi-scale and occlusion problem is an important evaluation criterion. Table 2 and Table 3 show the performance on scale subsets and occlusion subsets of Caltech, which confirms the better ability of our model to deal with multi-scale and occlusion.

RocScale is set as large and medium respectively in table 2. With the more challenging metric, most of methods exhibit dramatic performance drops while our model become the best with MR of $0.9 \%$ and $41.2 \%$ respectively.

The closest competitors have the MR of $2.4 \%$ from Checkerboards under large scale and $56.3 \%$ from CCF under medium scale. Remarkably, our method has better property than other methods on multiscale pedestrian detection. 
Table 2. Comparison of our model with others on Caltech-USA Scale subsets.

\begin{tabular}{c|c|c|c}
\hline Method & $\begin{array}{c}\text { MR } \\
\text { RocScale } \\
\text { large })\end{array}$ & Method & MR \\
\hline $\begin{array}{c}\text { Multiple Fused CNNs } \\
\text { (ours) }\end{array}$ & $\mathbf{0 . 0 0 9 6 7 2}$ & $\begin{array}{c}\text { Multiple Fused CNNs } \\
\text { (ours) }\end{array}$ & $\mathbf{0 . 4 1 2 1 9 9}$ \\
\hline Checkerboards & 0.024231 & CCF & 0.56291 \\
\hline Checkerboards+ & 0.024231 & Deep Parts & 0.564207 \\
\hline CCF & 0.028844 & Checkerboards & 0.579635 \\
\hline Deep Cascade & 0.039644 & Checkerboards+ & 0.579635 \\
\hline Deep Cascade+ & 0.039644 & CCF+CF & 0.595553 \\
\hline CCF+CF & 0.042224 & SCCPriors & 0.61272 \\
\hline DeepParts & 0.043697 & LDCF & 0.618213 \\
\hline Katamari & 0.067506 & Katamari & 0.63135 \\
\hline TA-CNN & 0.070006 & SpatialPooling+ & 0.633776 \\
\hline
\end{tabular}

RocOcc is set as partial and heavy respectively in Table 3 to check out the models' property of dealing with occlusion. Our model has a MR of $20.2 \%$ under partial subsets, which is very close to the best of $19.9 \%$ from DeepParts. In addition, our model get $47.9 \%$ MR under heavy subsets, which is an improvement of $12.5 \%$ over DeepParts. Others get larger MR because of not good at dealing with the highly occluded pedestrians.

These comparisons demonstrate that our model has better performance on complex situations in multi-scale and occlusion.

Table 3. Comparison of our model with others on Caltech-USA Occlusion subsets

\begin{tabular}{c|c|c|c}
\hline Method & $\begin{array}{c}\text { MR } \\
\text { (RocOcc }=\text { partial) }\end{array}$ & Method & $\begin{array}{c}\text { MR } \\
\text { (Roc0cc = heavy) }\end{array}$ \\
\hline $\begin{array}{c}\text { Deep Parts } \\
\begin{array}{c}\text { Multiple Fused CNNs } \\
\text { (ours) }\end{array}\end{array}$ & $\mathbf{0 . 1 9 9 3 2 8}$ & Multiple Fused CNNs (ours) & $\mathbf{0 . 4 7 9 8 4 3}$ \\
\hline Checkerboards & 0.2019 & Deep Parts & 0.604151 \\
\hline Checkerboards+ & 0.313079 & TA-CNN & 0.703543 \\
\hline TA-CNN & 0.327993 & CCF & 0.724423 \\
\hline CCF+CF & 0.376901 & CCF+CF & 0.72683 \\
\hline SpatialPooling & 0.392483 & Checkerboards & 0.779349 \\
\hline SpatialPooling+ & 0.392483 & Checkerboards+ & 0.779349 \\
\hline CCF & 0.405743 & SpatialPooling & 0.782469 \\
\hline SCCPriors & 0.412883 & SpatialPooling+ & 0.782469 \\
\hline Speed & SDN & 0.787738 \\
\hline
\end{tabular}

Speed comparison. To more adapt the practical applications, calculation speed is a crucial factor for pedestrian detection.

As shown in Table 4, our method is the most competitive one on efficiency competed with others, which achieves $8 \mathrm{fps}$ on GTX Titan X. This efficiency is about 4 times faster than RPN+BF and CompACT-Deep. This is because our model has a simple structure without any resampling phase or use of artificial features. We believe the efficiency and simplicity of our model will benefit practical applications. 
Table 4. Comparison with other methods on speed

\begin{tabular}{c|c|c|c}
\hline Method & Hardware (GPU) & $\begin{array}{c}\text { seconds per } \\
\text { image }\end{array}$ & fps \\
\hline Multiple Fused & Titan X (Pascal) & 0.12 & 8 \\
CNNs & & & \\
\hline RPN+BF & K40 Z & 0.5 & 2 \\
SAF R-CNN & Titan X (Pascal) & 0.59 & 1.7 \\
CompACT-Deep & K40 Z & 0.5 & 2 \\
F-DNN & Titan X (Pascal) & 0.16 & 6.2 \\
\hline
\end{tabular}

\section{Conclusion}

These are some clear examples in Figure 5. We selected some interesting examples from image test and video test with different scenarios. We can see from the Figure that our model perform very well in a variety of scenarios.
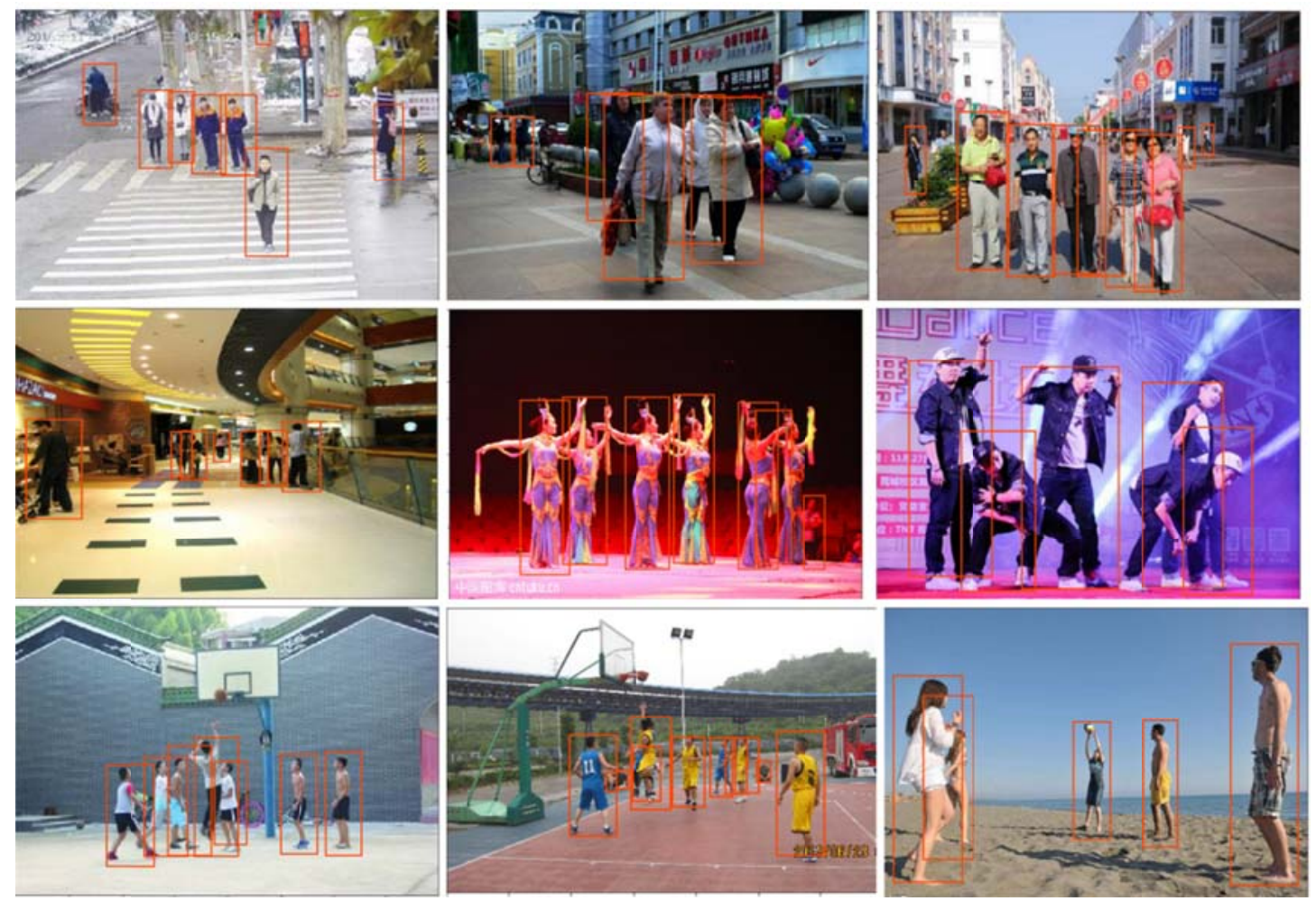

Figure 6. Detections of our model.

We proposed a pedestrian detector named Multiple Fused CNNs, which not only keeps a competitive quality of pedestrian detection, but obviously accelerate the calculation speed. The key point of the model is to make full use of the semantic features of different convolutional layers by detecting on multi-scale fused feature maps. This model does not need any artificial features.

The model is simpler and faster than other models. Thus, it could be easier to put it into practical systems such as driver assistance systems and intelligent monitoring systems.

\section{References}

[1] N. Dalal and W. Triggs, "Histograms of Oriented Gradients for Human Detection," in CVPR, 
2004.

[2] T. Ojala, M. Pietika"inen, and T. Ma"enpa"a", "Multiresolution gray-scale and rotation invariant texture classification with local binary patterns," PAMI, vol. 24, no. 7, pp. 971-987, 2002.

[3] Dollar P, Appel R, Belongie S, et al. Fast Feature Pyramids for Object Detection[J]. IEEE Transactions on Pattern Analysis \& Machine Intelligence, 2014, 36(8):1532-1545.

[4] Benenson R, Omran M, Hosang J, et al. Ten Years of Pedestrian Detection, What Have We Learned?[J]. 2014, 8926:613-627.

[5] Wanli Ouyang and Xiaogang Wang. Joint deep learning for pedestrian detection [C]. IEEE International Conference on Computer Vision, 2013, pp.2056-2063.

[6] Ping Luo, Yonglong Tian, Xiaogang Wang, and Xiaoou Tang. Switchable deep network for pedestrian detection [C]. IEEE Conference on Computer Vision and Pattern Recognition, 2014, pp. 899-906.

[7] J. Hosang, M. Omran, R., and B. Schiele, "Taking a Deeper Look at Pedestrians," in CVPR, 2015.

[8] Girshick R, Donahue J, Darrell T,et al. Rich feature hierarchies for accurate object detection and semantic segmentation[C]//Computer Vision and Pattern Recognition (CVPR), 2014 IEEE Conference on. IEEE,2014:580-587.

[9] Girshick R. Fast R-CNN[J]. Computer Science, 2015.

[10] Ren S, He K, Girshick R, et al. Faster R-CNN: Towards Real-Time Object Detection with Region Proposal Networks.[J]. IEEE Transactions on Pattern Analysis \& Machine Intelligence, 2016:1-1.

[11] Tian Y, Luo P, Wang X, et al. Deep Learning Strong Parts for Pedestrian Detection[C]// IEEE International Conference on Computer Vision. IEEE, 2015:1904-1912.

[12] Zhang L, Lin L, Liang X, et al. Is Faster R-CNN Doing Well for Pedestrian Detection?[M]// Computer Vision - ECCV 2016. 2016.

[13] Redmon J, Divvala S, Girshick R, et al. You Only Look Once: Unified, Real-Time Object Detection[J]. Computer Science, 2016:779-788.

[14] Liu W, Anguelov D, Erhan D, et al. SSD: Single Shot MultiBox Detector[J]. 2015.

[15] Hariharan B, Arbeláez P, Girshick R, et al. Hypercolumns for Object Segmentation and Fine-grained Localization[J]. 2014:447-456.

[16] Liu W, Rabinovich A, Berg A C. ParseNet: Looking Wider to See Better[J]. Computer Science, 2015.

[17] Bell S, Zitnick C L, Bala K, et al. Inside-Outside Net: Detecting Objects in Context with Skip Pooling and Recurrent Neural Networks[J]. 2015:2874-2883.

[18] Lin T Y, Dollár P, Girshick R, et al. Feature Pyramid Networks for Object Detection[J]. 2016.

[19] Chen, L.C., Papandreou, G., Kokkinos, I.,Murphy, K., Yuille, A.L.: Semantic image segmentation with deep convolutionalnets and fully connected crfs. In: ICLR. (2015)

[20] Dollar P, Wojek C, Schiele B, et al. Pedestrian detection: A benchmark[J]. Proc Cvpr, 2009:304-311. 